\title{
Common Fixed Point Results for Compatible Map in Digital Metric Space
}

\author{
Sumitra Dalal'1, Ibtisam A. Masmali'1, Ghaliah Y. Alhamzi² \\ ${ }^{1}$ Jazan University, Jazan, KSA \\ ${ }^{2} \mathrm{Al}$ Imam Mohammad Ibn Saud Islamic University, Riyadh, KSA \\ Email: mathsqueen-d@yahoo.com, ibtisam234@hotmail.com, Gyalhamzi@imamu.edu.sa
}

How to cite this paper: Dalal, S., Masmali, I.A. and Alhamzi, G.Y. (2018) Common Fixed Point Results for compatible Map in Digital Metric Space. Advances in Pure Mathematics, 8, 362-371. https://doi.org/10.4236/apm.2018.83019

Received: January 20, 2018

Accepted: March 26, 2018

Published: March 29, 2018

Copyright (C) 2018 by authors and Scientific Research Publishing Inc. This work is licensed under the Creative Commons Attribution International License (CC BY 4.0).

http://creativecommons.org/licenses/by/4.0/

\begin{abstract}
In this paper, we prove common fixed point results for quadruple self-mappings satisfying an implicit function which is general enough to cover a multitude of known as well as unknown contractions. Our results modify, unify, extend and generalize many relevant results existing in literature. Also, we define the concept of compatible maps and its variants in the setting of digital metric space and establish some common fixed point results for these maps. Also, an application of the proposed results is quoted in this note.
\end{abstract}

\section{Keywords}

Implicit Relation, Compatible Maps, Fixed Point Results, Digital Metric Space, Metric Space

\section{Introduction}

Digital topology is an emerging area based on general topology and functional analysis and focuses on studying digital topological properties of $n$-dimensional digital spaces, where as Euclidean topology deals with topological properties of subspaces of the $n$-dimensional real space, which has contributed to the study of some areas of computer sciences such as computer graphics, image processing, approximation theory, mathematical morphology, optimization theory etc. Rosenfield [1] was the first to consider digital topology as a tool to study digital images. Boxer [2] produced the digital versions of the topological concepts and later studied digital continuous functions [3]. Ege and Karaca [4] [5] established relative and reduced Lefschetz fixed point theorem for digital images and proposed the notion of a digital metric space and proved the famous Banach Contraction Principle for digital images.

Fixed point theory leads to lots of applications in mathematics, computer 
science, engineering, game theory, fuzzy theory, image processing and so forth. In metric spaces, this theory begins with the Banach fixed-point theorem which provides a constructive method of finding fixed points and an essential tool for solution of some problems in mathematics and engineering and consequently has been generalized in many ways. Up to now, several developments have occurred in this area. A major shift in the arena of fixed point theory came in 1976 when Jungck [6] [7] [8], defined the concept of commutative and compatible maps and proved the common fixed point results for such maps. Later on, Sessa [9] gave the concept of weakly compatible, and proved results for set valued maps. Certain altercations of commutativity and compatibility can also be found in [10] [11] [12] [13].

In 2014, He et al. [14] proved the common fixed points for pair of weak commutative mappings on a complete multiplicative metric spaces as follow:

Theorem 1. Let $S, T, A$ and $B$ be mappings of a complete multiplicative metric space $(X, d)$ into itself satisfying the following conditions:

1) $S(X) \subseteq B(X), T(X) \subseteq A(X)$,

2) $d(S x, T y)[\max =\{d(A x, B y), d(S x, T y), d(S x, A x)$,

$$
d(B y, T y), d(S x, B y), d(A x, T y)\}]^{\lambda},
$$

for all $x, y \in X, \lambda \in\left(0, \frac{1}{2}\right)$.

3) one of the mappings $S, T, A$ and $B$ is continuous. Assume that the pairs $(A, S)$ and $(B, T)$ are weakly commuting.Then $S, T, A$ and $B$ have a unique common fixed point.

The objective of this paper is to give digital version of above theorem using an implicit function which is general enough to cover several linear as well as some nonlinear contractions. Our results generalize and extend the many results existing in literature.

This paper is organized as follows. In the first part, we give the required background about the digital topology and fixed point theory. In the next section, we state and prove main results for compatible mappings and compatible mappings of types (A) and (P) in digital metric spaces. Our results improve and generalize many other results existing in literature. Finally, we give an important application of fixed point theorem to digital images. Lastly, we make some conclusions.

\section{Preliminaries}

Let $X$ be subset of $Z^{n}$ for a positive integer $n$ where $Z^{n}$ is the set of lattice points in the $n$-dimensional Euclidean space and $\rho$ represent an adjacency relation for the members of $X$. A digital image consists of $(X, \rho)$.

Definition 2. [15] Let $l, n$ be positive integers, $1 \leq l \leq n$ and two distinct points $a=\left(a_{1}, a_{2}, \cdots, a_{n}\right), \quad b=\left(b_{1}, b_{2}, \cdots, b_{n}\right) \in Z^{n}, a$ and $b$ are $k_{l}$-adjacent if there are at most 1 indices $i$ such that 


$$
\left|a_{i}-b_{i}\right|=1
$$

and for all other indices $j$ such that

$$
\left|a_{j}-b_{j}\right| \neq 1, \quad a_{j}=b_{j} .
$$

A $\rho$-neighbour of $a \in Z^{n}$ is a point of $Z^{n}$ that is $\rho$-adjacent to a where $\rho=\{2,4,6,8,18,26\}$ and $n \in\{1,2,3\}$. The set $N_{p}(a)=\{b / b$ is $\rho$-adjacent to $a\}$ is called the $\rho$-neighbourhood of a. A digital interval is defined by

$$
[p, q]_{z}=\{z \in Z / p \leq z \leq q\} \text {, where } p, q \in Z \text { and } p<q .
$$

A digital image $X \subset Z^{n}$ is $\rho$-connected if and only if for every pair of different points $u, v \in X$, there is a set $\left\{u_{0}, u_{1}, \cdots, u_{r}\right\}$ of points of digital image $X$ such that $u=u_{0}, v=u_{r}$ and $u_{i}$ and $u_{i+1}$ are $\rho$-neighbour where $i=0,1,2, \cdots, r-1$.

Definition 3. [15] Let $\left(X, \rho_{0}\right) \subset Z^{n_{0}},\left(Y, \rho_{1}\right) \subset Z^{n_{1}}$ be digital images and $T: X \rightarrow Y$ be a function, then

1) $T$ is said to be $\left(\rho_{0}, \rho_{1}\right)$-continuous, if for all $\rho_{0}$-connected subset $E$ of $X$, $f(E)$ is a $\rho_{1}$-connected subset of $Y$.

2) For all $\rho_{0}$-adjacent points $\left\{u_{0}, u_{1}\right\}$ of $X$, either $T\left(u_{0}\right)=T\left(u_{1}\right)$ or $T\left(u_{0}\right)$ and $T\left(u_{1}\right)$ are a $\rho_{1}$-adjacent in $Y$ if and only if $T$ is $\left(\rho_{0}, \rho_{1}\right)$ -continuous.

3) If $T$ is $\left(\rho_{0}, \rho_{1}\right)$-continuous, bijective and $T^{-1}$ is $\left(\rho_{0}, \rho_{1}\right)$-continuous, then $T$ is called $\left(\rho_{0}, \rho_{1}\right)$-isomorphism and denoted by $X \cong_{\left(\rho_{0}, \rho_{1}\right)} Y$.

Definition 4. [16] A sequence $\left\{X_{n}\right\}$ of points of a digital metric space $(X, d, \rho)$ is a Cauchy sequence if for all $\varepsilon>0$, there exists $\delta \in N$ such that for all $n, m>\delta$, then $d\left(x_{n}, x_{m}\right)<\varepsilon$.

Definition 5. [16] A sequence $\left\{x_{n}\right\}$ of points of a digital metric space $(X, d, \rho)$ converges to a limit $p \in X$ if for all $\varepsilon>0$, there exists $\delta \in \mathbb{N}$ such that for all $n>\delta$, then $d\left(x_{n}, p\right)<\varepsilon$.

Definition 6. [16] $A$ digital metric space $(X, d, \rho)$ is a complete digital metric space if any Cauchy sequence $\left\{x_{n}\right\}$ of points of $(X, d, \rho)$ converges to a point $p$ of $(X, d, \rho)$.

Definition 7. [16] Let $(X, d, \rho)$ be any digital metric space and $T:(X, d, \rho) \rightarrow(X, d, \rho)$ be a self digital map. If there exists $\alpha \in(0,1)$ such that for all $x \in X, d\left(T_{x}, T_{y}\right) \leq \alpha(x, y)$ then $T$ is called a digital contraction map.

Proposition 8. [16] Every digital contraction map is digitally continuous.

Theorem 9. [16] (Banach Contraction principle) Let $(X, d, \rho)$ be a complete digital metric space which has a usual Euclidean metric in $Z^{n}$. Let, $T: X \rightarrow X$ be a digital contraction map. Then $T$ has a unique fixed point, i.e. there exists a unique $p \in X$ such that $T(p)=p$.

\section{Main Results}

Definition 10. [17] Suppose that $(X, d, \rho)$ is a complete digital metric space 
and $S, T: X \rightarrow X$ be maps defined on $X$. Then $S$ and $T$ are said to be commutative if

$$
S \circ T=T \circ S, \quad \forall x \in X .
$$

Definition 11. [17] The self maps $S$ and $T$ of a digital metric space $(X, d, \rho)$ are said to be weakly commutative if

$$
d(S(T(x)), T(S(x))) \leq(S(x), T(x)) \text { for all } x \in X .
$$

Remark 1. [18] Every pair of commutative maps is weakly commutative but the converse is not true.

Definition 12. [19] [20] Let $S$ and $T$ be self maps of a digital metric space $(X, d, \rho)$ and $\left\{x_{n}\right\}$ is a sequence in $X$ such that

$$
\lim _{n \rightarrow \infty} S\left(x_{n}\right)=\lim _{n \rightarrow \infty} T\left(X_{n}\right)=t \quad \text { for some } t \text { in } X .
$$

Then

1) $S$ and $T$ and are said to be compatible if $\lim _{n \rightarrow \infty} d\left(S T x_{n}, T S x_{n}\right)=0$

2) $S$ and $T$ and are said to be compatible of type $(A)$ if $\lim _{n \rightarrow \infty} d\left(S T x_{n}, T T x_{n}\right)=0$ and $\lim _{n \rightarrow \infty} d\left(T S x_{n}, S S x_{n}\right)=0$

3) $S$ and $T$ and are said to be compatible of type $(P)$ if $\lim _{n \rightarrow \infty} d\left(S S x_{n}, T T x_{n}\right)=0$

4) $\bar{O}$-compatible if for any sequence $\left\{x_{n}\right\} \subset X$ with $S x_{n} \uparrow t$ and $T x_{n} \uparrow t$ (for some $t \in X$ ) implies $\lim _{n \rightarrow \infty} d\left(S T x_{n}, T S x_{n}\right)=0$,

5) $\underline{O}$-compatible if for any sequence $\left\{x_{n}\right\} \subset X$ with $S x_{n} \downarrow t$ and $T x_{n} \downarrow t$ (for some $t \in X$ ) implies $\lim _{n \rightarrow \infty} d\left(S T x_{n}, T S x_{n}\right)=0$,

6) O-compatible if for any sequence $\left\{x_{n}\right\} \subset X$ with $S x_{n} \downarrow t$ and $T x_{n} \uparrow t$ (for some $t \in X$ ) implies $\lim _{n \rightarrow \infty} d\left(S T x_{n}, T S x_{n}\right)=0$.

7) weakly compatible if $S(T x)=T(S x), \forall x \in X$.

Remark 2. [19] [20] In a digital metric space, compatibility $\Rightarrow$ O-compatibility $\Rightarrow \bar{O}$-compatibility (as well as $\underline{O}$-compatibility) $\Rightarrow$ weak compatibility.

Definition 13. [19] [20] An ordered metric space $(X, d)$ is called O-complete (resp. $\bar{O}$-complete, $\underline{O}$-complete) if every Cauchy sequence converges in $X$.

Remark 3. In a digital metric space, completeness $\Rightarrow O$-completeness $\Rightarrow \bar{O}$ -completeness (as well as $\underline{O}$-completeness).

Remark 4. Every pair of weakly commuting maps is compatible but the converse is not true.

Definition 14. [19] [20] Let $(T, S)$ be a pair of self-mappings on a metric space $(X, d)$ and $x \in X$. We say that $T$ is $S$-continuous at $X$ if for any sequence $\left\{x_{n}\right\} \subset X, S x_{n} \stackrel{d}{\longrightarrow} S X \Rightarrow T x_{n} \stackrel{d}{\longrightarrow} T X$. Moreover, $T$ is called $S$-continuous if it is $S$-continuous at every point of $X$.

Notice that, on setting $S=I X$, Definition 14 reduces to the usual definition 
of continuity.

Definition 15. [20] Let $(T, S)$ be a pair of self-mappings on a metric space $(X, d)$ and $x \in X$. Then $T$ is called $(S, O)$-continuous (resp. $(S, \bar{O})$ -continuous, $(S, \underline{O})$-continuous) at $X$ if $T x_{n} \stackrel{d}{\longrightarrow} T X$, for every sequence $\left\{x_{n}\right\} \subset X$ with $S x_{n} \uparrow S x$ (resp. $S x_{n} \downarrow S x, S x_{n} \uparrow \downarrow S x$ ). Moreover, $T$ is called $(S, O)$-continuous (resp. $(S, \bar{O})$-continuous, $(S, \underline{O})$-continuous) if it is $(S, O)$-continuous (resp. $(S, \bar{O})$-continuous, $(S, \underline{O})$-continuous) at every point of $x \in X$.

Notice that, on setting $S=I X$, Definition 15 reduces to the usual definition of $O$-continuity (resp. $\bar{O}$-continuity, $\underline{O}$-continuity) of a self-mapping ( $T$ on $X)$.

Remark 5. In digital metric space, $S$-continuity $\Rightarrow(S, O)$-continuity $\Rightarrow$ $(S, \bar{O})$-continuity (as well as $(S, \underline{O})$-continuity).

Proposition 16. Let $S$ and $T$ be compatible maps of type $(A)$ on a digital metric space $(X, d, \rho)$. If one of $S$ and $T$ is continuous, then $S$ and $T$ are compatible.

Proof. Since $S$ and $T$ are compatible maps of type $(A)$, so $\lim _{n \rightarrow \infty} d\left(S T x_{n}, T T x_{n}\right)=0$ and $\lim _{n \rightarrow \infty} d\left(T S x_{n}, S S x_{n}\right)=0$, whenever $\left\{x_{n}\right\}$ is a sequence in $X$ such that

$$
\lim _{n \rightarrow \infty} S x_{n}=\lim _{n \rightarrow \infty} T x_{n}=t \quad \text { for some } t \text { in } X .
$$

Let $S$ is continuous, then

$$
\lim _{n \rightarrow \infty} S T x_{n} \rightarrow S t \quad \text { and } \quad \lim _{n \rightarrow \infty} S S x_{n} \rightarrow S t
$$

and hence

$$
d\left(S T x_{n}, T S x_{n}\right) \leq d\left(S T x_{n}, S S x_{n}\right)+d\left(S S x_{n}, T S x_{n}\right) .
$$

Letting $n \rightarrow \infty$, we have $\lim _{n \rightarrow \infty} d\left(S T x_{n}, T S x_{n}\right)=0$ implies $S$ and $T$ are compatible.

Proposition 17. Let $S$ and $T$ be compatible maps on a digital metric space $(X, d, \rho)$ into itself. Suppose that

$$
\lim _{n \rightarrow \infty} S x_{n}=\lim _{n \rightarrow \infty} T x_{n}=t \quad \text { for some } t \in X .
$$

Then

1) $\lim _{n \rightarrow \infty} S T x_{n}=T t$ if $T$ is continuous at $t$.

2) $\lim _{n \rightarrow \infty} T S x_{n}=S t$ if $S$ is continuous at $t$.

Proof.

1) Suppose that $T$ is continuous at $t$. Since $\lim _{n \rightarrow \infty} S x_{n}=\lim _{n \rightarrow \infty} T x_{n}=t$ for some $t \in X$, we have

$$
\lim _{n \rightarrow \infty} T S x_{n}=T t .
$$

Since $S$ and $T$ are compatible maps, we have

$$
d\left(S T x_{n}, T t\right) \leq d\left(S T x_{n}, T S x_{n}\right)+d\left(T S x_{n}, T t\right) \rightarrow 0
$$

as $n \rightarrow \infty$ and hence the proof. 
2) The proof of $\lim _{n \rightarrow \infty} T S x_{n}=S t$ follows by similar arguments as in (a).

Proposition 18. Let $S$ and $T$ be compatible maps on a digital metric space $(X, d, \rho)$ into itself. If $S t=T t$ for some $t \in X$, then

$$
S T t=T S t=S S t=T T t \text {. }
$$

\section{Implicit Relation and Related Concepts}

In recent years, Popa [21] have used implicit functions rather than contraction conditions to prove fixed point theorems in metric spaces whose strength lie in their unifying power, as an implicit function can cover several contraction conditions at the same time, which include known as well as some unknown contraction conditions. This fact is evident from examples furnished in Popa [21]. In this section, in order to prove our results, we define a set of suitable implicit functions involving six real non-negative arguments that was given in [22].

In the literature, there are several types of implicit contraction mappings where many nice consequences of fixed point theorems could be derived. First, denote $\Phi$ the set of functions $\phi:[0, \infty) \rightarrow[0, \infty)$ satisfying:

1) $\phi$ is nondecreasing,

2) $\sum_{n=0}^{\infty} \phi^{n}<\infty$ for each $t \in[0, \infty)$, where $\phi^{n}$ is the nth iterate of $\phi$.

Remark 6. It is easy to see that if $\phi \in \Phi$, then $\phi(0)=0$ and $\phi(t)<t$, . $\forall t>0$

Definition 19. [22] Let $R^{+}$denote the set of non-negative real numbers and let $\Gamma$ be the set of all continuous functions $\Gamma: R^{6} \rightarrow R$ satisfying the following conditions:

1) For each $u, v \geq 0, T(u, v, u, v, u+v, 0) \leq 0 \Rightarrow u \leq \phi(v)$.

2) $T(u, 0,0, u, u, 0)>0$ or $T(u, v, 0,0, v, u)>0$.

Example 20. $T\left(t_{1}, t_{2}, t_{3}, t_{4}, t_{5}, t_{6}\right)=t_{1}-\alpha \max \left\{t_{2}, t_{3}, t_{4}\right\}-(1-\alpha)\left[a t_{5}+b t_{6}\right]$ where $0 \leq \alpha \leq 1,0 \leq a<\frac{1}{2}, 0 \leq b<\frac{1}{2}$.

Example 21. $T\left(t_{1}, t_{2}, t_{3}, t_{4}, t_{5}, t_{6}\right)=t_{1}-k \max \left\{t_{2}, t_{3}, t_{4}, \frac{1}{2}\left[a t_{5}+b t_{6}\right]\right\}$, where $k \in(0,1)$.

Example 22. $T\left(t_{1}, t_{2}, t_{3}, t_{4}, t_{5}, t_{6}\right)=t_{1}-\phi \max \left\{t_{2}, t_{3}, t_{4}, \frac{1}{2}\left[a t_{5}+b t_{6}\right]\right\}$, where $\phi: R^{+} \rightarrow R^{+}$is right continuous and $\phi(0)=0$ and $\phi(t)<t, \forall t>0$.

Theorem 23. Let $A, B, S$ and $T$ be four self-mappings of a complete digital metric space $(X, d, \rho)$ satisfying the following conditions:

a) $S(X) \subset B(X)$ and $T(X) \subset A(X)$;

b) the pairs $(A, S)$ and $(B, T)$ are compatible;

c) one of $S, T, A$ and $B$ is continuous;

d) $F(d(A x, B y), d(S x, T y), d(A x, S x), d(B y, T y), d(A x, T y), d(B y, S x))$, 
$\forall x, y \in X$

Then $A, B, S$ and $T$ have a unique common fixed point in $X$.

Proof. Since $S(X) \subset B(X)$, we can consider a point $x_{0} \in X$, there exists $x_{1} \in X$ such that

$$
S x_{0}=B x_{1}=y_{0} .
$$

Also, for this point $x_{1}$, there exists $x_{2} \in X$ such that

$$
\operatorname{Tx}_{1}=A x_{2}=y_{1} \text {. }
$$

Continuing in this way, we can construct a sequence $\left\{y_{n}\right\}$ in $X$ such that

$$
y_{2 n}=S x_{2 n}=B x_{2 n+1}, y_{2 n+1}=T x_{2 n+1}=A x_{2 n+2} \quad \text { for each } n \geq 0 .
$$

Now, we have to show that $\left\{y_{n}\right\}$ is Cauchy sequence in $(X, d, \rho)$. Using condition (d), we have

$$
\begin{gathered}
F\left(d\left(A x_{2 n}, B x_{2 n+1}\right), d\left(S x_{2 n}, T x_{2 n+1}\right), d\left(A x_{2 n}, S x_{2 n}\right),\right. \\
\left.d\left(B x_{2 n+1}, T x_{2 n+1}\right), d\left(A x_{2 n}, T x_{2 n+1}\right), d\left(B x_{2 n+1}, S x_{2 n}\right)\right) \leq 0 \\
\text { i.e } F\left(d\left(y_{2 n-1}, y_{2 n}\right), d\left(y_{2 n}, y_{2 n+1}\right), d\left(y_{2 n-1}, y_{2 n}\right),\right. \\
\left.\quad d\left(y_{2 n}, y_{2 n+1}\right), d\left(x_{2 n-1}, y_{2 n+1}\right), d\left(y_{2 n}, y_{2 n}\right)\right) \leq 0,
\end{gathered}
$$

which is,

$$
\begin{aligned}
& F\left(d\left(y_{2 n-1}, y_{2 n}\right), d\left(y_{2 n}, y_{2 n+1}\right), d\left(y_{2 n-1}, y_{2 n}\right),\right. \\
& \left.d\left(y_{2 n}, y_{2 n+1}\right), d\left(y_{2 n-1}, y_{2 n}\right), d\left(y_{2 n}, y_{2 n+1}\right), 0\right) \leq 0,
\end{aligned}
$$

implies that

$$
d\left(y_{2 n}, y_{2 n+1}\right) \leq \phi\left(d\left(y_{2 n-1}, y_{2 n}\right)\right)<d\left(y_{2 n-1}, y_{2 n}\right) .
$$

Therefore, the sequence $\left\{d\left(y_{2 n}, y_{2 n+1}\right)\right\}$ is strictly decreasing. Then there exists $r \geq 0$ such that

$$
\lim _{n \rightarrow \infty} d\left(y_{2 n}, y_{2 n+1}\right)=r
$$

Suppose that $r>0$, then, letting $n \rightarrow \infty$ in above equation, $r \leq \phi(r)<r$, which is imposible. Hence $r=0$, that is,

$$
\lim _{n \rightarrow \infty} d\left(y_{2 n}, y_{2 n+1}\right)=0 .
$$

By the completeness of $X$, there exists $z \in X$ such that $y_{n} \rightarrow \infty$ as $n \rightarrow \infty$. Consequently, the subsequences $S x_{2 n}, A x_{2 n}, T x_{2 n+1}$ and $B x_{2 n+1}$ of $\left\{y_{n}\right\}$ also converge to a point $z \in X$. Now, suppose that $A$ is continuous.Then $\left\{A A x_{2 n}\right\}$ and $\left\{A S x_{2 n}\right\}$ converge to $A z$ as $n \rightarrow \infty$. Since the mappings $A$ and $S$ are compatible on $X$, it follows from Proposition 17 that $\left\{S A x_{2 n}\right\}$ converge to $A z$ as $n \rightarrow \infty$.

Now, we claim that $A z=z$. Consider,

$$
\begin{aligned}
& F\left(d\left(A A x_{2 n}, B x_{2 n+1}\right), d\left(S A x_{2 n}, T x_{2 n+1}\right), d\left(A A x_{2 n}, S A x_{2 n}\right),\right. \\
& \left.d\left(B x_{2 n+1}, T x_{2 n+1}\right), d\left(A A x_{2 n}, T x_{2 n+1}\right), d\left(B x_{2 n+1}, S A x_{2 n}\right)\right) \leq 0 .
\end{aligned}
$$


Letting $n \rightarrow \infty$ we have

$$
\begin{aligned}
& F(d(A z, z), d(A z, z), d(A z, A z), d(z, z), d(A z, z), d(z, A z)) \leq 0 \\
& \Rightarrow F(d(A z, z), d(A z, z), 0,0, d(A z, z), d(z, A z)) \leq 0
\end{aligned}
$$

yields $A z=z$. Again, from (d), we obtain

$$
\begin{aligned}
& F\left(d\left(A z, B x_{2 n+1}\right), d\left(S z, T x_{2 n+1}\right), d(A z, S z),\right. \\
& \left.d\left(B x_{2 n+1}, T x_{2 n+1}\right), d\left(A z, T x_{2 n+1}\right), d\left(B x_{2 n+1}, S z\right)\right) \leq 0 .
\end{aligned}
$$

Letting $n \rightarrow \infty$ we get

$$
\begin{aligned}
& F(d(z, z), d(S z, z), d(z, S z), d(z, z), d(z, z), d(z, S z)) \leq 0 \\
& =F(0, d(S z, z), d(z, S z), 0,0, d(z, S z)) \leq 0 \Rightarrow S z=z .
\end{aligned}
$$

Since $S(X) \subset B(X)$, there exists $u \in X$ such that

$$
z=A z=S z=B u \text {. }
$$

By using $(\mathrm{d})$, we can obtain

$$
\begin{aligned}
& F(d(A z, B u), d(S z, T u), d(A z, S z), d(B u, T u), d(A z, T u), d(B u, S z)) \leq 0 \\
& =F(d(z, z)), d(z, T u), d(z, z), d(z, T u), d(z, T u), d(z, z)) \leq 0 . \\
& =F(0, d(z, T u), 0, d(z, T u), d(z, T u), 0) \leq 0 \Rightarrow z=T u .
\end{aligned}
$$

As $B$ and $T$ are compatible on $X$ and $z=T u=B u$, by Proposition 18, we have $T B u=B T u$ and hence $B z=T B u=B T u=T z$. Also, we have

$$
\begin{aligned}
& F(d(A z, B z), d(S z, T z), d(A z, S z), d(B z, T z), d(A z, T z), d(B z, S z)) \leq 0 . \\
& \Rightarrow F(0, d(z, T z), 0,0, d(z, T z), d(T z, z)) \leq 0 \Rightarrow z=T z .
\end{aligned}
$$

Thus $z=T z=B z$ and so $z$ is a common fixed point of $A, B, S$ and $T$. Similarly, we can use above assertion in case of continuity of $B$ or $S$ or $T$ and uniqueness of the common fixed point follows directly from the condition (d) and hence the proof follows.

Theorem 24. Let $A, B, S$ and $T$ be four self-mappings of a complete digital metric space $(X, d, \rho)$ satisfying the conditions $(\mathrm{a}),(\mathrm{c})$ and $(\mathrm{d})$. If the pairs $(A, S)$ and $(B, T)$ are compatible of type $(\mathrm{A})$ or of type $(\mathrm{P})$ then $A, B, S$ and $T$ have a unique common fixed point in $X$.

Proof. Theorem is direct consequence of Proposition 16 and Theorem 23.

Example 25. Let $X=[1, \infty)$ with usual metric and $A, B, S, T: X \rightarrow X$ be self mappings such that

$$
\begin{gathered}
T(x)=\left\{\begin{array}{ll}
2 x-1, & x \leq 2 \\
3, & x>2
\end{array}, A(x)= \begin{cases}3 x-2, & x \leq 2 \\
x, & x>2\end{cases} \right. \\
B(x)=\left\{\begin{array}{ll}
x, & x \leq 2 \\
x+1, & x>2
\end{array}, S(x)=1 .\right.
\end{gathered}
$$

Then we see that $S(X) \subset B(X)$ and $T(X) \subset A(X)$ and if we consider a sequence $\left\langle x_{n}\right\rangle=1+\frac{1}{n}$, then 


$$
\lim _{n \rightarrow \infty} d\left(A S x_{n}, S A x_{n}\right)=0 \text { and } \lim _{n \rightarrow \infty} d\left(B T x_{n}, T B x_{n}\right)=0
$$

implies that the pairs $(A, S)$ and $(B, T)$ are compatible and $S$ is continuous. Also the condition (d) holds. Hence all the conditions of Theorem 23 are satisfied and $x=1$ is common fixed point of the mappings.

\section{Applications of Common Fixed Point Theorems in Digital Metric Space}

In this section, we give an application of digital contractions to solve the problem related to image compression. The aim of image compression is to reduce redundant image information in the digital image. When we store an image we may come across certain type of problems like either memory data is usually too large or stored image has not more information than original image. Also, the quality of compressed image can be poor. For this reason, we must pay attention to compress a digital image. Fixed point theorem can be used for image compression of a digital image.

\section{Conclusions}

The purpose of this paper is four fold which can be described as follows.

1) We slightly modify the implicit relation of Popa so that contraction conditions obtained involving functional inequalities.

2) Proved some common fixed point theorems by using modified implicit relation, compatibility and its variants in the setting of digital-metric spaces.

3) Generalized the Theorem 1 and many others too existing in literature, derived related results and furnished illustrative examples.

4) Our results generate scope for other researchers to prove the results by utilizing other contractions, weak compatibility, control function and admissible maps in a digital framework.

\section{References}

[1] Rosenfeld, A. (1979) Digital Topology. The American Mathematical Monthly, 86, 621-630. https://doi.org/10.2307/2321290

[2] Boxer, L. (2005) Properties of Digital Homotopy. Journal of Mathematical Imaging and Vision, 22, 19-26. https://doi.org/10.1007/s10851-005-4780-y

[3] Boxer, L. (2010) Continuous Maps on Digital Simple Closed Curves. Applied Mathematics, 1, 377-386. https://doi.org/10.4236/am.2010.15050

[4] Ege, O. and Karaca, I. (2013) Lefschetz Fixed Point Theorem for Digital Images. Fixed Point Theory and Applications, 2013, 13 pages.

[5] Ege, O. and Karaca, I. (2014) Applications of The Lefschetz Number to Digital Images. Bulletin of the Belgian Mathematical Society-Simon Stevin, 21, 823-839.

[6] Jungck, G. (1976) Commuting Mappings and Fixed Point. The American Mathematical Monthly, 83, 261-263. https://doi.org/10.2307/2318216

[7] Jungck, G. (1986) Compatible Mappings and Common Fixed Points. International Journal of Mathematics and Mathematical Sciences, 9, 771-779. 


\section{https://doi.org/10.1155/S0161171286000935}

[8] Jungck, G. (1996) Common Fixed Points for Noncontinuous Nonself Maps on Nonmetric Spaces. Far East Journal of Mathematical Sciences, 4, 199-215.

[9] Sessa, S. and Fisher, B. (1986) On Common Fixed Points of Weakly Commuting Mappings and Set-Valued Mappings. International Journal of Mathematics and Mathematical Sciences, 9, 323-329. https://doi.org/10.1155/S0161171286000406

[10] Das, K.M. and Naik, K.V. (1979) Common Fixed-Point Theorems for Commuting Maps on a Metric Space. Proceedings of the American Mathematical Society, 77, 369-373.

[11] Sumitra (2013) Coupled Fixed Point Theorems for Compatible Maps and Its Variants in Fuzzy Metric Spaces. Jordan Journal of Mathematics and Statistics (JJMS), 6, 141-155.

[12] Sumitra (2012) Coupled Fixed Point Theorem for Weakly Compatible Mappings in Intuitionistic Fuzzy Metric Spaces. International Journal of Computer Applications (0975-8887), 50.

[13] Žikić-Došenović, T. (2009) A Common Fixed Point Theorem for Compatible Mappings in Fuzzy Metric Spaces Using Implicit Relation. Acta Mathematica Hungarica, 125, 357-368. https://doi.org/10.1007/s10474-009-9029-6

[14] He, X., Song, M. and Chen, D. (2014) Common Fixed Points for Weak Commutative Mappings on a Multiplicative Metric Space. Fixed Point Theory and Applications, 2014, 48. https://doi.org/10.1186/1687-1812-2014-48

[15] Boxer, L. (1991) A Classical Constructions for the Digital Fundamental Group. Journal of Mathematical Imaging and Vision, 10, 51-62. https://doi.org/10.1023/A:1008370600456

[16] Ege, O. and Karaca, I. (2015) Banach Fixed Point Theorem for Digital Images. Journal of Nonlinear Sciences and Applications, 8, 237-245.

https://doi.org/10.22436/jnsa.008.03.08

[17] Jain, D. and Upadhaya, A.C. (1982) Weakly Commuting Mappings in Digital Metric Spaces. International Advanced Research Journal in Science, Engineering and Technology, 4, 771-831.

[18] Rani, A., Jyoti, K. and Rani, A. (2016) Common Fixed Point Theorems in Digital Metric Spaces. International Journal of Scientific \& Engineering Research, 7.

[19] Alam, A., Khan, Q.H. and Imdad, M. (2015) Enriching Some Recent Coincidence Theorems for Nonlinear Contractions in Ordered Metric Spaces. Fixed Point Theory and Applications, 2015, 141.

[20] Alam, A. and Imdad, M. (2015) Comparable Linear Contractions in Ordered Metric Spaces. https://arxiv.org/abs/1507.08987

[21] Popa, V.A. (1978) Fixed Point Theorem for Mapping in D-Complete Topological Spaces. Mathematica Moravica, 3, 43-48.

[22] Altun, I and Simsek, H. (2010) Some Fixed Point Theorems on Ordered Metric Spaces and Application. Fixed Point Theory and Applications, 2010, Article ID: 621492. 